\title{
Localization Error Estimate for the Massless Relativistic Kinetic Energy Operator
}

\author{
J-M. Barbaroux ${ }^{1}$, V. Vougalter ${ }^{2}$, S. Vugalter ${ }^{3}$ \\ 1 Aix-Marseille Université, CNRS, CPT, UMR 7332, 13288 Marseille, France \\ and Université de Toulon, CNRS, CPT, UMR 7332, 83957 La Garde, France. \\ ${ }^{2}$ University of Toronto, Department of Mathematics, Toronto, Ontario, M5S 2E4, Canada. \\ ${ }^{3}$ Karlsruhe Institute of Technology, Englerstrasse 2, 76131 Karlsruhe, Germany.
}

\begin{abstract}
We derive an estimate for the localization error of the relativistic kinetic operator $|\nabla|$ for massless particles in three dimensional configuration space. As a consequence, we obtain a localization estimate arbitrarily small in term of the localized kinetic energies.
\end{abstract}

Keywords and phrases: Localization error, relativistic kinetic energy.

Mathematics Subject Classification: $81 \mathrm{~V} 10$

\section{Introduction}

Geometric methods in multi-particle systems are based on partitions of unity of the configuration space $X$. These methods are very successful in studying bound states for multi-particle systems. Given a partition of unity $\left(w_{i}\right)_{i}$ of $L^{2}(X)$ with $w_{i} \in C^{2}(X ;[0,1])$ and $\sum_{i}\left(w_{i}\right)^{2}=1$, the localization formula for Schrödinger operators $H=-\Delta+V$ is

$$
H-\sum_{i} w_{i} H w_{i}=-\sum_{i}\left|\nabla w_{i}\right|^{2}
$$

where the term on the right hand side is called localization error term.

The identity (1.1) reduces the study of $H$ on the whole configuration space to the study of localized operators $w_{i} H w_{i}$, if one can derive good estimates for the localization error term.

The first result based on partition of unity of the configuration space was established at the beginning of 1960's. A localization formula for Laplace operator appeared for the first time, at least implicitly, in [5]. In [19] and later in [14], some types of localization error estimates were used to prove the so-called HVZ theorem.

Simple scaling arguments show that if the size of the region where we make a localization, namely the region where the supports of the functions $w_{i}$ overlap, is proportional to $R$, then the localization error is proportional to $1 / R^{2}$ in the case of Laplace kinetic energy. The HVZ theorem requires rather rough estimates on localization error, and the above argument is sufficient.

*Corresponding author. E-mail: barbarou@univ-tln.fr, Semjon.Wugalter@kit.edu, vitali@math.toronto.edu 
In the context of the study of discrete spectrum, refined estimates on the localization error are necessary. In [15] and [20] such refined estimates were established. What was observed in [20], is that for arbitrary small $\epsilon$, it is possible to construct localization functions $w_{i}(i=1,2)$ for separation of two regions such that the localization error in one of the regions is $\epsilon / R^{2}$ and in the other region is $c(\epsilon) / R^{2}$.

In [9] and [10], localization formula was rediscovered. It was applied to systems with potential having two centers in [9], and to the study of molecules in Born-Oppenheimer approximation in [10].

The next step was done in [16], [17] and [18]. There, the study of multi-particle systems with resonances at the bottom of the essential spectrum required a much better estimate of the localization error. It was proved in [17] and [18] that given $\epsilon>0$, using localization "inside" a region of radius $a / R$ for $w_{1}$ (in the sense that $w_{1}(x)=1$ for $|x| \leq a / R$ ) and "outside" a ball of radius $b / R$ for $w_{2}$ (in the sense $w_{2}(x)=1$ for $|x| \geq b / R$ ), then the localization error is $\epsilon / R^{2}$ assuming that the ratio $a / b$ is sufficiently small.

A systematical use of nonrelativistic localization formula was done to derive spectral properties of bound states of multi-particle Schrödinger operators by several authors, see e.g. [13], [8] and [12] and references therein.

Similarly to the Schrödinger case, to apply geometric method to relativistic many-body operators, the derivation of a relativistic localization formula was necessary. Such an identity was derived in [7] for the study of stability and instability of relativistic matter, yielding the following localization formula for the operator $|\nabla|$,

$$
\begin{aligned}
& \langle\psi,|\nabla| \psi\rangle-\left\langle w_{1} \psi,|\nabla| w_{1} \psi\right\rangle-\left\langle w_{2} \psi,|\nabla| w_{2} \psi\right\rangle \\
& =-\frac{1}{2 \pi^{2}} \sum_{i=1}^{2} \int_{\mathbb{R}^{3} \times \mathbb{R}^{3}} \frac{\psi(x) \overline{\psi(y)}}{|x-y|^{4}}\left(w_{i}(x)-w_{i}(y)\right)^{2} \mathrm{~d} x \mathrm{~d} y,
\end{aligned}
$$

for nonnegative $w_{1}$ and $w_{2}$ in $C^{2}\left(\mathbb{R}^{3} ;[0,1]\right)$ such that $w_{1}^{2}+w_{2}^{2}=1$. The right hand side of $(1.2)$ is called relativistic localization error.

Subsequently, this formula was applied in various cases to multi-particle models with relativistic particles, in order to derive estimates on the localization error (see [6], [2], [3], [4], [1] and [11] and references therein).

In the work at hand we derive an estimate for the relativistic localization error term in the case of relativistic massless particles. We show that the localization error for $|\nabla|$ can be controlled by an arbitrary small part of the kinetic energy.

\section{Main result}

Theorem 2.1. Pick arbitrary numbers $a>0, \epsilon \in\left(0,3^{-3 / 2}\right)$ and $\kappa \in \mathbb{R}$. Then, for $\gamma=\epsilon^{2 / 3}$ a, there exist $b>a$ and two real spherically symmetric functions $v_{1}(x), v_{2}(x) \in C^{2}\left(\mathbb{R}^{3}\right)$, such that for $w_{i}(x):=v_{i}(\alpha x)$ $(i=1,2)$, we have for all $\alpha$ small enough

$-w_{1}(x)^{2}+w_{2}(x)^{2}=1$

- $w_{1}(x)=1$ for $|x| \leq \frac{a}{\alpha}$ and $w_{1}(x)=0$ for $|x| \geq \frac{b}{\alpha}$,

and for all $\psi \in W^{1,2}\left(\mathbb{R}^{3}\right)$

$$
\begin{aligned}
& \left|\langle\psi,|\nabla| \psi\rangle-\left\langle w_{1} \psi,|\nabla| w_{1} \psi\right\rangle-\left\langle w_{2} \psi,|\nabla| w_{2} \psi\right\rangle\right| \\
& \leq \epsilon\left(\frac{\alpha}{a}\right)^{1-\kappa}\left\|\mathbf{1}\left(|x| \leq \frac{\gamma}{\alpha}\right)(1+|x|)^{-\kappa / 2} \psi\right\|^{2}+\epsilon\left\|\mathbf{1}\left(|x| \geq \frac{\gamma}{\alpha}\right)|x|^{-\frac{1}{2}} \psi\right\|^{2},
\end{aligned}
$$

where $\mathbf{1}(A)$ denotes the characteristic function of the set $A$.

Corollary 2.2. Pick arbitrary numbers $a>0$ and $\epsilon \in\left(0,3^{-3 / 2}\right)$. Then, there exist $b>$ a and two real spherically symmetric functions $v_{1}(x), v_{2}(x) \in C^{2}\left(\mathbb{R}^{3}\right)$ such that for $w_{i}(x):=v_{i}(\alpha x)(i=1,2)$, we have for all $\alpha$ small enough 
$-w_{1}(x)^{2}+w_{2}(x)^{2}=1$

- $w_{1}(x)=1$ for $|x| \leq \frac{a}{\alpha}$ and $w_{1}(x)=0$ for $|x| \geq \frac{b}{\alpha}$,

and for all $\psi \in W^{1,2}\left(\mathbb{R}^{3}\right)$

$$
\langle\psi,|\nabla| \psi\rangle \geq(1-\epsilon)\left\langle w_{1} \psi,|\nabla| w_{1} \psi\right\rangle+(1-\epsilon)\left\langle w_{2} \psi,|\nabla| w_{2} \psi\right\rangle .
$$

Remark 2.3. The proof of Theorem 2.1 can be adapted so that for arbitrary $b>0$ and $\epsilon \in\left(0,3^{-3 / 2}\right)$, one can find $a \in(0, b)$, and two real spherically symmetric functions $v_{1}(x), v_{2}(x) \in C^{2}\left(\mathbb{R}^{3}\right)$, such that for $w_{i}(x):=v_{i}(\alpha x)$, we have for $\alpha$ small enough, $w_{1}(x)^{2}+w_{2}(x)^{2}=1, w_{1}(x)=1$ for $|x| \leq \frac{a}{\alpha}$ and $w_{1}(x)=0$ for $|x| \geq \frac{b}{\alpha}$, and inequality (2.2) holds.

Proof of Corollary 2.2. Kato's inequality yields

$$
\frac{\pi}{2}\langle\psi,|\nabla| \psi\rangle \geq\left\langle\psi, \frac{1}{|x|} \psi\right\rangle=\left\langle w_{1} \psi, \frac{1}{|x|} w_{1} \psi\right\rangle+\left\langle w_{2} \psi, \frac{1}{|x|} w_{2} \psi\right\rangle
$$

Since for all $\gamma>0$ and $\alpha>0$ we have

$$
\left\|\mathbf{1}\left(|x| \leq \frac{\gamma}{\alpha}\right)(1+|x|)^{-\kappa / 2} \psi\right\|^{2}+\left\|\mathbf{1}\left(|x| \geq \frac{\gamma}{\alpha}\right)|x|^{-\frac{1}{2}} \psi\right\|^{2} \leq 2\left\langle\psi, \frac{1}{|x|} \psi\right\rangle,
$$

then, applying theorem 2.1 with $\kappa=1$, and using the above inequalities (2.3) and (2.4) yields the result.

Proof of Theorem 2.1. According to Lieb-Yau in [7, Theorem 9], the localization error for the operator $|\nabla|$ is given by $(1.2)$.

Our goal is to show that the functions $w_{1}(x), w_{2}(x)$ and the numbers $b$ and $\gamma$ can be chosen so that the r.h.s. of (2.1) is greater than the absolute value of (1.2).

We take $\tilde{v}_{1}($.$) a real function from [0, \infty)$ to $[0,1]$, with $\tilde{v}_{1}(r)=1$ for $r<a$, strictly decreasing in some interval $[a, a+\delta], \delta \in(0, a)$, in such a manner that

$$
\lim _{r \rightarrow a+0} \frac{\tilde{v}_{1}^{\prime}(r)^{2}}{1-\tilde{v}_{1}(r)^{2}}=0 .
$$

Let $\tilde{v}_{2}($.$) be the real function from [0, \infty)$ to $[0,1]$ such that $\tilde{v}_{1}^{2}+\tilde{v}_{2}^{2}=1$, and let us define $\tilde{w}_{i}(r):=$ $\tilde{v}_{i}(\alpha r)$.

Pick $\epsilon_{1}>0$. Therefore, using $(2.5)$ and the identity $\tilde{w}_{2}=\left(1-\tilde{w}_{1}^{2}\right)^{\frac{1}{2}}$, we can pick $\delta \in(0, a)$ independent of $\alpha$ and sufficiently small such that for any $r \in\left[\frac{a}{\alpha}, \frac{a+\delta}{\alpha}\right]$ holds

$$
\tilde{w}_{1}^{\prime}(r)^{2}+\tilde{w}_{2}^{\prime}(r)^{2} \leq \tilde{w}_{1}^{\prime}(r)^{2}+\frac{\tilde{w}_{1}^{\prime}(r)^{2}}{1-\tilde{w}_{1}(r)^{2}} \leq \epsilon_{1} \alpha^{2}
$$

We define, for $i=1,2$,

$$
w_{i}(x):=\tilde{w}_{i}(|x|) \quad \text { and } \quad v_{i}(x):=\tilde{v}_{i}(|x|) .
$$

Because of symmetry in $x$ and $y$, we can write

$$
\begin{aligned}
& \sum_{i=1}^{2} \int \frac{\psi(x) \overline{\psi(y)}}{|x-y|^{4}}\left(w_{i}(x)-w_{i}(y)\right)^{2} \mathrm{~d} x \mathrm{~d} y \\
& =2 \operatorname{Re} \sum_{i=1}^{2} \int_{|x| \leq|y|} \frac{\psi(x) \overline{\psi(y)}}{|x-y|^{4}}\left(w_{i}(x)-w_{i}(y)\right)^{2} \mathrm{~d} x \mathrm{~d} y=2\left(I_{1}+I_{2}+I_{3}\right),
\end{aligned}
$$

where, for $\gamma \in(0, a)$ : 
$-I_{1}$ is the integral over the region

$$
\left\{(x, y) \in \mathbb{R}^{3} \times \mathbb{R}^{3}\left|\frac{\gamma}{\alpha} \leq\right| x|\leq| y|,| y \mid \leq \frac{a+\delta}{\alpha}\right\} .
$$

$-I_{2}$ is the integral over the region

$$
\left\{(x, y) \in \mathbb{R}^{3} \times \mathbb{R}^{3}\left|\frac{\gamma}{\alpha} \leq\right| x|\leq| y|,| y \mid>\frac{a+\delta}{\alpha}\right\} .
$$

$-I_{3}$ is the integral over the region

$$
\left\{(x, y) \in \mathbb{R}^{3} \times \mathbb{R}^{3}|| x\left|\leq \frac{\gamma}{\alpha},\right| y \mid \geq \frac{a}{\alpha}\right\} .
$$

- On the remaining region

$$
\left\{(x, y) \in \mathbb{R}^{3} \times \mathbb{R}^{3}|| x\left|\leq \frac{\gamma}{\alpha},\right| y\left|<\frac{a}{\alpha},\right| x|\leq| y \mid\right\},
$$

the integral is zero, because $w_{i}(x)-w_{i}(y)=0$ in this region.

- Estimate of $I_{1}$. For $|x|<|y| \leq \frac{a+\delta}{\alpha}$ the inequality (2.6) implies

$$
\begin{aligned}
\sum_{i=1}^{2}\left(w_{i}(x)-w_{i}(y)\right)^{2} & \leq \max _{|\widetilde{x}| \leq \frac{a+\delta}{\alpha}}\left(\tilde{w}_{1}^{\prime}(|\widetilde{x}|)^{2}+\frac{\tilde{w}_{1}^{\prime}(|\widetilde{x}|)^{2}}{1-\tilde{w}_{1}^{2}(|\widetilde{x}|)}\right)|x-y|^{2} \\
& \leq \epsilon_{1} \alpha^{2}|x-y|^{2}
\end{aligned}
$$

Moreover,

$$
\left\{\frac{\gamma}{\alpha} \leq|x| \leq|y|,|y| \leq \frac{a+\delta}{\alpha}\right\} \subset\left\{\frac{\gamma}{\alpha} \leq|x| \leq \frac{2 a}{\alpha}, \frac{\gamma}{\alpha} \leq|y| \leq \frac{2 a}{\alpha}\right\} .
$$

Therefore since the set in the right hand side of (2.9) is symmetric in $x$ and $y$, then with (2.8) and (2.9), we obtain

$$
\begin{aligned}
\left|I_{1}\right| & \leq \epsilon_{1} \alpha^{2} \int_{|y| \leq \frac{a+\delta}{\alpha} ; \frac{\gamma}{\alpha} \leq|x| \leq|y|} \frac{|\psi(x)||\psi(y)|}{|x-y|^{2}} \mathrm{~d} x \mathrm{~d} y \\
& \leq \epsilon_{1} \alpha^{2} \int_{|x-y| \leq \frac{3 a}{\alpha}} \frac{\mathrm{d}(x-y)}{|x-y|^{2}} \int_{\frac{\gamma}{\alpha} \leq|x| \leq \frac{2 a}{\alpha}}|\psi(x)|^{2} \mathrm{~d} x \\
& \leq 12 \pi \epsilon_{1} \alpha a\|\psi(x)\|_{\frac{\gamma}{\alpha} \leq|x| \leq \frac{2 a}{\alpha}}^{2} \\
& \leq 24 \pi \epsilon_{1} a^{2}\left\|\mathbf{1}\left(|x| \geq \frac{\gamma}{\alpha}\right)|x|^{-1 / 2} \psi(x)\right\|^{2} .
\end{aligned}
$$

Taking $\epsilon_{1}=\epsilon /\left(96 \pi a^{2}\right)$, we arrive at

$$
\left|I_{1}\right| \leq \frac{\epsilon}{4}\left\|\mathbf{1}\left(|x| \geq \frac{\gamma}{\alpha}\right)|x|^{-1 / 2} \psi(x)\right\|^{2} .
$$

- Estimate of $I_{2}$. We note that $\tilde{w}_{1}\left(\frac{a+\delta}{\alpha}\right) \leq 1$, and for $|y|>\frac{a+\delta}{\alpha},|y| \geq|x|$, we have

$$
\begin{aligned}
\left(w_{2}(x)-w_{2}(y)\right)^{2} & =\left(\sqrt{1-w_{1}^{2}(x)}-\sqrt{1-w_{1}^{2}(y)}\right)^{2} \\
& =\left(\frac{\left(w_{1}(x)-w_{1}(y)\right)\left(w_{1}(x)+w_{1}(y)\right)}{\sqrt{1-w_{1}^{2}(x)}+\sqrt{1-w_{1}^{2}(y)}}\right)^{2} \\
& \leq \frac{4}{1-\tilde{w}_{1}^{2}\left(\frac{a+\delta}{\alpha}\right)}\left(w_{1}(x)-w_{1}(y)\right)^{2} \\
& =C_{0}\left(w_{1}(x)-w_{1}(y)\right)^{2}
\end{aligned}
$$


with $C_{0}$ independent of $\alpha$ (since $\left.\tilde{w}_{1}\left(\frac{a+\delta}{\alpha}\right)=\tilde{v}_{1}(a+\delta)\right)$. The last inequality implies

$$
\left|I_{2}\right| \leq\left(C_{0}+1\right) \int_{\frac{\gamma}{\alpha} \leq|x|<|y| ;|y|>\frac{a+\delta}{\alpha}} \frac{|\psi(x)||\psi(y)|}{|x-y|^{4}}\left(w_{1}(x)-w_{1}(y)\right)^{2} \mathrm{~d} x \mathrm{~d} y .
$$

At this point, we specify the function $w_{1}$ for $|x| \in\left[\frac{a+\delta}{\alpha}, \frac{b}{\alpha}\right]$, and we set

$$
w_{1}(x)=\ln \left(\frac{\alpha|x|}{b}\right) \ln ^{-1}\left(\frac{a+\delta}{b}\right) \tilde{w}_{1}\left(\frac{a+\delta}{\alpha}\right) .
$$

It is easy to see that for $|x| \in\left[\frac{a+\delta}{\alpha}, \frac{b}{\alpha}\right]$,

$$
\left|w_{1}(x)-w_{1}(y)\right| \leq \ln ^{-1}\left(\frac{b}{a+\delta}\right)\left|\ln \left(\frac{|x|}{|y|}\right)\right| \leq \ln ^{-1}\left(\frac{b}{a+\delta}\right) \frac{|y-x|}{|x|} .
$$

Let $\lambda>0$ be a constant which we specify later. We split the region corresponding to $I_{2}$ into $\Omega_{1} \cup \Omega_{2}$, where

$$
\begin{aligned}
& \Omega_{1}=\left\{(x, y)\left|\frac{\gamma}{\alpha}<\right| x|<| y|,| y\left|>\frac{a+\delta}{\alpha},\right| x-y|>\lambda| x \mid\right\}, \\
& \Omega_{2}=\left\{(x, y)\left|\frac{\gamma}{\alpha}<\right| x|<| y|,| y\left|>\frac{a+\delta}{\alpha},\right| x-y|\leq \lambda| x \mid\right\} .
\end{aligned}
$$

Let us first discuss the contribution from $\Omega_{1}$. One has

$$
\begin{aligned}
& \int_{\Omega_{1}} \frac{|\psi(x)||\psi(y)|}{|x-y|^{4}}\left(w_{1}(x)-w_{1}(y)\right)^{2} \mathrm{~d} x \mathrm{~d} y \leq \int_{\Omega_{1}} \frac{|\psi(x)||\psi(y)|}{|x-y|^{4}} \mathrm{~d} x \mathrm{~d} y \\
& \leq\left(\int_{\Omega_{1}} \frac{|\psi(x)|^{2}}{|x-y|^{4}} \mathrm{~d} x \mathrm{~d} y\right)^{1 / 2}\left(\int_{\Omega_{1}} \frac{|\psi(y)|^{2}}{|x-y|^{4}} \mathrm{~d} x \mathrm{~d} y\right)^{1 / 2}
\end{aligned}
$$

and

$$
\begin{aligned}
\int_{\Omega_{1}} \frac{|\psi(x)|^{2}}{|x-y|^{4}} \mathrm{~d} x \mathrm{~d} y & \leq \int_{|x|>\frac{\gamma}{\alpha}}|\psi(x)|^{2}\left(\int_{|x-y|>\lambda|x|} \frac{\mathrm{d} y}{|x-y|^{4}}\right) \mathrm{d} x \\
& \leq \frac{4 \pi}{\lambda}\left\|\mathbf{1}\left(|x| \geq \frac{\gamma}{\alpha}\right)|x|^{-\frac{1}{2}} \psi\right\|^{2} .
\end{aligned}
$$

Since $|x|<\frac{|x-y|}{\lambda}$ by definition of $\Omega_{1}$, it implies that $|y| \leq|y-x|+|x|<\frac{1+\lambda}{\lambda}|x-y|$,

$$
\begin{aligned}
\int_{\Omega_{1}} \frac{|\psi(y)|^{2}}{|x-y|^{4}} \mathrm{~d} x \mathrm{~d} y & \leq \int_{|y|>\frac{\gamma}{\alpha}}|\psi(y)|^{2}\left(\int_{|x-y|>\frac{\lambda}{1+\lambda}|y|} \frac{\mathrm{d} x}{|x-y|^{4}}\right) \mathrm{d} y \\
& \leq 4 \pi \frac{\lambda+1}{\lambda}\left\|\mathbf{1}\left(|x| \geq \frac{\gamma}{\alpha}\right)|x|^{-\frac{1}{2}} \psi\right\|^{2}
\end{aligned}
$$

One obtains

$$
\begin{aligned}
\int_{\Omega_{1}} \frac{|\psi(x)||\psi(y)|}{|x-y|^{4}} & \left(w_{1}(x)-w_{1}(y)\right)^{2} \mathrm{~d} x \mathrm{~d} y \\
& \leq 4 \pi \frac{\sqrt{\lambda+1}}{\lambda}\left\|\mathbf{1}\left(|x| \geq \frac{\gamma}{\alpha}\right)|x|^{-\frac{1}{2}} \psi\right\|^{2}
\end{aligned}
$$

from the combination of (2.15), (2.16) and (2.14). 
Next, we focus on the contributions from the region $\Omega_{2}=\Omega_{2}^{\prime} \cup \Omega_{2}^{\prime \prime}$, where

$$
\begin{aligned}
& \Omega_{2}^{\prime}=\left\{(x, y)\left|\frac{\gamma}{\alpha} \leq\right| x|\leq| y|,| y\left|>\frac{a+\delta}{\alpha},\right| x-y|\leq \lambda| x|,| x \mid \geq \frac{a+\delta}{\alpha}\right\}, \\
& \Omega_{2}^{\prime \prime}=\left\{(x, y)\left|\frac{\gamma}{\alpha} \leq\right| x|\leq| y|,| y\left|>\frac{a+\delta}{\alpha},\right| x-y|\leq \lambda| x|,| x \mid<\frac{a+\delta}{\alpha}\right\} .
\end{aligned}
$$

From (2.13), since $|x| \geq(a+\delta) / \alpha$ in $\Omega_{2}^{\prime}$, we obtain

$$
\begin{aligned}
& \int_{\Omega_{2}^{\prime}} \frac{|\psi(x)||\psi(y)|}{|x-y|^{4}}\left(w_{1}(x)-w_{1}(y)\right)^{2} \mathrm{~d} x \mathrm{~d} y \\
& \leq \ln ^{-2}\left(\frac{b}{a+\delta}\right)\left(\int_{\Omega_{2}^{\prime}} \frac{|\psi(x)|^{2}}{|x-y|^{2}|x|^{2}} \mathrm{~d} x \mathrm{~d} y\right)^{\frac{1}{2}}\left(\int_{\Omega_{2}^{\prime}} \frac{|\psi(y)|^{2}}{|x-y|^{2}|x|^{2}} \mathrm{~d} x \mathrm{~d} y\right)^{\frac{1}{2}} \\
& \left.\left.\leq \ln ^{-2}\left(\frac{b}{a+\delta}\right)\left(\int_{|x|>\frac{\gamma}{\alpha}} \frac{|\psi(x)|^{2}}{|x|^{2}}\left(\int_{|x-y|<\lambda|x|} \frac{\mathrm{d} y}{|x-y|^{2}}\right) \mathrm{d} x\right)^{\frac{1}{2}} \frac{\mathrm{d} x}{|x-y|^{2}}\right) \mathrm{~d} y\right)^{\frac{1}{2}} \\
& \quad \times\left(( 1 + \lambda ) ^ { 2 } \int _ { | y | > \frac { \gamma } { \alpha } } \frac { | \psi ( y ) | ^ { 2 } } { | y | ^ { 2 } } \left(\int_{|x-y|<\lambda|y|}\right.\right. \\
& \leq 4 \pi \lambda(1+\lambda) \ln ^{-2}\left(\frac{b}{a+\delta}\right)\left\|\mathbf{1}\left(|x| \geq \frac{\gamma}{\alpha}\right)|x|^{-\frac{1}{2}} \psi\right\|^{2},
\end{aligned}
$$

where we have used that $|y| \leq|x-y|+|x| \leq(1+\lambda)|x|$ so that $\frac{1}{|x|^{2}}<\frac{(1+\lambda)^{2}}{|y|^{2}}$, and $|x-y| \leq \lambda|x|<\lambda|y|$ on $\Omega_{2}^{\prime}$.

We choose $\lambda$ large enough, independently of $\alpha$, to fulfill the inequality

$$
4 \pi \frac{\sqrt{\lambda+1}}{\lambda}\left(\frac{4}{1-\tilde{w}_{1}^{2}\left(\frac{a+\delta}{\alpha}\right)}+1\right)<\frac{\epsilon}{4}
$$

and subsequently, for that given $\lambda$, we choose $b$ sufficiently large that

$$
4 \pi \lambda \ln ^{-2}\left(\frac{b}{a+\delta}\right)\left(\frac{4}{1-\tilde{w}_{1}^{2}\left(\frac{a+\delta}{\alpha}\right)}+1\right)<\frac{\epsilon}{4},
$$

which shows together with (2.18)

$$
\int_{\Omega_{2}^{\prime}} \frac{|\psi(x)||\psi(y)|}{|x-y|^{4}}\left(w_{1}(x)-w_{1}(y)\right)^{2} \mathrm{~d} x \mathrm{~d} y \leq \frac{\epsilon}{4}\left\|\mathbf{1}\left(|x| \geq \frac{\gamma}{\alpha}\right)|x|^{-\frac{1}{2}} \psi\right\|^{2} .
$$

We next estimate the contribution of $\Omega_{2}^{\prime \prime}$. For any $\epsilon_{2}>0$, one can pick $b$ large enough (independently on $\alpha$ ) so that the derivative of $w_{1}$ is bounded by $\epsilon_{2} \alpha$. Moreover, we remark that on $\Omega_{2}^{\prime \prime}$, one has $|x-y| \leq \lambda|x|<\lambda \frac{a+\delta}{\alpha}$ and $|y|<|x|+|x-y|<(1+\lambda)|x|<(1+\lambda) \frac{a+\delta}{\alpha}$. Thus,

$$
\begin{aligned}
& \int_{\Omega_{2}^{\prime \prime}} \frac{|\psi(x)||\psi(y)|}{|x-y|^{4}}\left(w_{1}(x)-w_{1}(y)\right)^{2} \mathrm{~d} x \mathrm{~d} y \\
& \leq \epsilon_{2}^{2} \alpha^{2} \int_{\frac{\gamma}{\alpha}<|x|<\frac{a+\delta}{\alpha}}\left(\int_{\frac{a+\delta}{\alpha}<|y|<(\lambda+1) \frac{a+\delta}{\alpha},|x-y|<\lambda \frac{a+\delta}{\alpha}} \frac{|\psi(x)||\psi(y)|}{|x-y|^{2}} \mathrm{~d} y\right) \mathrm{d} x \\
& \leq \epsilon_{2}^{2} \alpha^{2}\left(\int_{|x-y|<\lambda \frac{a+\delta}{\alpha}} \frac{\mathrm{d}(x-y)}{|x-y|^{2}}\right)\left\|\mathbf{1}\left(\frac{\gamma}{\alpha} \leq|x| \leq \frac{a+\delta}{\alpha}\right) \psi(x)\right\| \\
& \quad \times\left\|\mathbf{1}\left(\frac{a+\delta}{\alpha} \leq|y| \leq(\lambda+1) \frac{a+\delta}{\alpha}\right) \psi(y)\right\| \\
& \leq 4 \pi \epsilon_{2}^{2}(a+\delta)^{2} \lambda \sqrt{1+\lambda}\left\|\mathbf{1}\left(|x| \geq \frac{\gamma}{\alpha}\right)|x|^{-\frac{1}{2}} \psi(x)\right\|^{2}
\end{aligned}
$$


where we have used Young's inequality, and the fact that $\gamma<a$. Thus taking $\epsilon_{2}<\min \left\{\epsilon, 1 /\left(16 \pi \epsilon_{2}^{2}(a+\right.\right.$ $\left.\left.\delta)^{2} \lambda \sqrt{1+\lambda}\right)\right\}$, gives

$$
\begin{aligned}
& \int_{\Omega_{2}^{\prime \prime}} \frac{|\psi(x)||\psi(y)|}{|x-y|^{4}}\left(w_{1}(x)-w_{1}(y)\right)^{2} \mathrm{~d} x \mathrm{~d} y \\
& \leq \epsilon / 4|| \mathbf{1}\left(|x| \geq \frac{\gamma}{\alpha}\right)|x|^{-\frac{1}{2}} \psi(x) \|^{2} .
\end{aligned}
$$

The inequalities (2.21) and (2.23) thus give

$$
\left|I_{2}\right| \leq \frac{\epsilon}{2}\left\|\mathbf{1}\left(|x| \geq \frac{\gamma}{\alpha}\right)|x|^{-\frac{1}{2}} \psi(x)\right\|^{2} .
$$

Let us sum up the choices of the constants so far. Given $\epsilon>0$ and $a>0$, we have first chosen $\epsilon_{1}=\epsilon /\left(96 \pi a^{2}\right)$ and then picked $\delta$ small enough depending on $\epsilon$ so that (2.6) holds. With these choices, we derived the estimate (2.10) for $I_{1}$. Then, for $\lambda$ given by (2.19), we have taken $\epsilon_{2}$ small enough so that $4 \pi \epsilon_{2}(a+\delta)^{2} \lambda \sqrt{\lambda+1}<1$, and then chosen $b$ large enough so that both (2.20) holds and the derivative of $w_{1}$ is bounded by $\epsilon_{2} \alpha$. This yielded the estimate for $I_{2}$.

Therefore, (2.10) and (2.24) allows one to conclude that

$$
\left|I_{1}\right|+\left|I_{2}\right| \leq \frac{3 \epsilon}{4}\left\|\mathbf{1}\left(|x| \geq \frac{\gamma}{\alpha}\right)|x|^{-\frac{1}{2}} \psi\right\|^{2} .
$$

- Estimate of $I_{3}$. Assuming $\gamma \in\left(0, \frac{a}{3}\right)$, we have $|x-y| \geq \frac{2 a}{3 \alpha} \geq \frac{a}{3 \alpha}+|x|$ in the integration domain of $I_{3}$. Thus, for $\nu \in(0,2)$, we can write

$$
\left|I_{3}\right| \leq 2^{3+\nu} \int_{|x|<\frac{\gamma}{\alpha}} \frac{|\psi(x)|}{\left(\frac{a}{3 \alpha}+|x|\right)^{2-\nu}} \mathrm{d} x \int_{|y|>\frac{a}{\alpha}} \frac{|\psi(y)|}{|y|^{2+\nu}} \mathrm{d} y .
$$

For all $\kappa \in \mathbb{R}$, one has

$$
\begin{aligned}
& \left(\int_{|x|<\frac{\gamma}{\alpha}} \frac{|\psi(x)| \mathrm{d} x}{\left(\frac{a}{3 \alpha}+|x|\right)^{2-\nu}}\right)^{2} \\
& \leq\left(\int_{|x| \leq \frac{\gamma}{\alpha}} \frac{|\psi(x)|^{2} \mathrm{~d} x}{\left(\frac{a}{3 \alpha}+|x|\right)^{\kappa}}\right) \int_{|x| \leq \frac{\gamma}{\alpha}} \frac{\mathrm{d} x}{\left(\frac{a}{3 \alpha}+|x|\right)^{4-2 \nu-\kappa}} \\
& \leq c\left(\frac{\alpha}{a}\right)^{4-\kappa-2 \nu}\left(\frac{\gamma}{\alpha}\right)^{3}\left\|\mathbf{1}\left(|x| \leq \frac{\gamma}{\alpha}\right)(1+|x|)^{-\frac{\kappa}{2}} \psi\right\|^{2},
\end{aligned}
$$

where $c$ is a constant independent on $\alpha$ and $\kappa$. The second integral on the r.h.s. of (2.26) can be controlled by

$$
\begin{aligned}
\int_{|y|>\frac{a}{\alpha}} \frac{|\psi(y)|}{|y|^{2+\nu}} \mathrm{d} y & \leq\left(\int_{|y|>\frac{a}{\alpha}} \frac{|\psi(y)|^{2}}{|y|} \mathrm{d} y\right)^{\frac{1}{2}}\left(\int_{|y|>\frac{a}{\alpha}} \frac{\mathrm{d} y}{|y|^{3+2 \nu}}\right)^{\frac{1}{2}} \\
& \leq c\left(\frac{\alpha}{a}\right)^{\nu} \| \mathbf{1}\left(|y| \geq \frac{a}{\alpha}|y|^{-\frac{1}{2}} \psi(y) \| .\right.
\end{aligned}
$$

Collecting (2.26)-(2.28) yields

$$
\begin{aligned}
\left|I_{3}\right| \leq \frac{2 c \alpha^{1-\kappa}}{\epsilon} \frac{\gamma^{3}}{a^{4-\kappa}} \| \mathbf{1}(|x| & \left.\leq \frac{\gamma}{\alpha}\right)(1+|x|)^{-\kappa / 2} \psi \|^{2} \\
+\frac{\epsilon}{4} \| \mathbf{1}(|x| & \left.>\frac{a}{\alpha}\right)|x|^{-\frac{1}{2}} \psi \|^{2} .
\end{aligned}
$$

Picking $\gamma$ small enough independently of $\alpha$, using inequalities (2.25) and (2.29), and noting that the functions $w_{1}$ and $w_{2}$ can easily be approximated by functions in $C^{2}\left(\mathbb{R}^{3}\right)$ without changing the above estimates, one obtains the desired localization estimate (2.1).

Acknowledgements. J.-M.B was supported by the project SQFT ANR-12-JS01-0008-01. 


\section{References}

[1] J.-M. Barbaroux, T. Chen, S. Vugalter, Binding conditions for atomic $N$-electron systems in non-relativistic QED, Ann. Henri Poincaré Vol. 4, no.6, (2003),1101-1136

[2] R. Benguria, H. Siedentop, E. Stockmeyer, Dissociation of homonuclear relativistic molecular ions, Ann. Henri Poincaré Vol. 2, no.1, (2001), 27-40.

[3] H.L. Cycon, R.G. Froese, W. Kirsch, B. Simon, Schrödinger Operators with Application to Quantum Mechanics and Global Geometry, Springer-Verlag, Berlin (1987).

[4] M. Griesemer, E.H. Lieb, M. Loss, Ground states in non-relativistic quantum electrodynamics, Invent. Math. Vol. 145 (2001), 557-595.

[5] R.S. Ismagilov, Conditions for the semiboundedness and discreteness of the spectrum in the case of one-dimensional differential operators, Dokl. Akad. Nauk SSSR Vol. 140 (1961), 33-36

[6] R. Lewis, H. Siedentop, S. Vugalter, The essential spectrum of relativistic multi-particle operators, Ann. Inst. H. Poincaré Phys. Théor. Vol. 67 no.1, (1997), 1-28.

[7] E. H. Lieb, H.-T. Yau, The stability and instability of relativistic matter, Comm. Math. Phys. Vol. 118 (1988), $177-213$.

[8] E.H. Lieb, I.M. Sigal, B. Simon, W. Thirring, Approximate neutrality of large Z-ions, Comm. Math. Phys. Vol. 116, (1988), 635-644.

[9] J.D. Morgan, Schrödinger operators whose potentials have separated singularities, J. Operator Theory Vol. 1, (1979), 109-115.

[10] J.D. Morgan, B. Simon, On the asymptotics of Born-Oppenheimer curves for large nuclear separation Int. J. Quantum Chem. Vol. 17, (1980), 1143-1166.

[11] S. Morozov, S. Vugalter, Stability of atoms in the Brown- Ravenhall model, Ann. Henri Poincaré Vol. 7, no.4, (2006), 661-687.

[12] M.B. Ruskai, Improved estimate of the number of bound states of negatively charged bosonic atoms, Ann. Inst. H. Poincaré Phys. Théor. Vol. 61, no.2, (1994), 153-162.

[13] I.M. Sigal, Geometric methods in the quantum many-body problem. Nonexistence of very negative ions, Comm. Math. Phys. Vol. 85 no.2, (1982), 309-324.

[14] A.G. Sigalov, I.M. Sigal, Invariant description, with respect to transpositions of identical particles, of the energy operator spectrum of quantum-mechanical systems, Teor. Mat. Fiz. Vol. 5 no.1, (1970), 73-93.

[15] J. Uchiyama, Finiteness of the number of discrete eigenvalues of the Schrödinger operator for a three particule system Publ. RIMS, Kyoto Univ. Vol. 5, (1969), 51-63.

[16] S.A. Vugalter, G.M. Zhislin, The symmetry of Efimov's effect in systems of three-quantum particles Comm. Math. Phys. Vol. 87 no.1, (1982/83), 89-103.

[17] S.A. Vugalter, G.M. Zhislin, On the finiteness of discrete spectrum in the $n$-particle problem, Rep. Math. Phys. Vol. 19 no. 1, (1984), 39-90.

[18] S.A. Vugalter, G.M. Zhislin, Trudy Moskov. Mat. Obshch. Vol. 49 (1986), 95-112.

[19] G.M. Zhislin, A study of the spectrum of the Schrödinger operator for a system of several particles, Trudy Moskov. Mat. Obshch. Vol. 9 (1960), 81-120.

[20] G.M. Zhislin, Finiteness of the discrete spectrum in the quantum problem of $n$ particles, Teoret. Mat. Fiz. Vol. 21 $(1974), 60-73$. 